\title{
Quilopericárdio idiopático
}

\author{
Fábio Fernandes, Edmundo Arteaga, Mauro Sérgio Stateri Carvalho, Barbara Maria lanni, \\ Paulo Pego Fernandes, Charles Mady
}

São Paulo, SP

\begin{abstract}
Descrevemos um caso de derrame pericárdico idiopático em que, durante investigação diagnóstica, foi constatado ser um quilopericárdio. A linfografia mostrou aplasia parcial do ducto torácico. Realizamos uma breve revisão da etiopatogenia, quadro clínico, métodos diagnósticos complementares e possibilidades terapêuticas do quilopericárdio.
\end{abstract}

\section{Idiopathic chylopericardium}

We describe a case of a patient with idiopathic pericardial effusion that during investigation proved to be a chylopericardium. Lymphangiography showed the chylopericardium to be due to partial aplasia of the thoracic duct. A brief review the etiology, clinical feature, diagnostic procedures and therapeutic possibilities of chylopericardium is presented.

Arq Bras Cardiol, volume 71 (n 2), 131-134, 1998

O quilopericárdio é uma condição clínica infreqüente, como etiologia de derrame pericárdico, geralmente associado a traumatismos, pós-operatório de cirurgias torácicas, tumores mediastinais ou idiopático ${ }^{1}$. Até 1997 , somente 89 casos de quilopericárdio idiopático foram descritos na literatura mundial ${ }^{2}$. A fisiopatologia em muitos casos é obscura, porém, a linfografia, em alguns casos, estabelece a origem do quilopericárdio, como sendo uma anormalidade do sistema linfático. Descrevemos um caso de quilopericárdio idiopático decorrente de aplasia parcial do ducto torácico e realizamos a revisão de literatura.

\section{Relato do caso}

Paciente de 19 anos de idade, natural e proveniente de São Paulo, Capital, encaminhado ao Instituto do Coração com diagnóstico de derrame pericárdico de etiologia indefinida, diagnosticada há dois anos, após episódio gripal, tendo realizado estudo radiológico do tórax, que evidenciou área cardíaca aumentada (fig. 1). O ecocardiograma confirmou derrame pericárdico importante sem sinais de restrição diastólica. Durante esse período manteve-se assintomático do ponto de vista cardiovascular, em acompanhamento clínico ambulatorial. Investigação inicial mostrou fator

Instituto do Coração do Hospital das Clínicas - FMUSP

Correspondência: Fabio Fernandes - Incor - Av. Dr. Enéas C. Aguiar, 44 - $05403-$ 000 - São Paulo, SP

Recebido para publicação em 11/3/98

Aceito em 26/5/98 antinúcleo, células LE, fator reumatóide, sorologia para HIV e sífilis negativos, assim como hormônios tireoidianos normais. Feita hipótese diagnóstica de derrame pericárdico idiopático.

Internado em setembro/97 para elucidação diagnóstica; o paciente foi submetido em 18/9/97 a pericardioscopia com biópsia. Durante o procedimento também foi realizada pericardiectomia ântero-lateral direita com vídeoassistência e drenagem pleural à direita em virtude de abertura da pleura. À abertura do saco pericárdico houve saída de $300 \mathrm{~mL}$ de líquido de aspecto branco leitoso, sugerindo origem quilosa. A biópsia pericárdica mostrou pericardite crônica discreta, com fibrose, neovascularização e reatividade de células mesoteliais. A cultura do líquido foi negativa. Feita hipótese diagnóstica de quilopericárdio.

Em vista dessa descoberta foi iniciada a investigação da etiologia do quilopericárdio, sendo realizados os seguintes exames: linfocintilografia com macroagregados de albumina marcados com tecnécio ${ }^{99}$, que evidenciou volumosa coleção linfática intra-torácica (quilotórax) e interrupção do ducto torácico ao nível abdominal (fig. 2). Com esse resultado, realizou-se linfografia com injeção de contraste em ductos coletores da região dorsal de ambos os pés. Observou-se aspecto conservado dos ductos linfáticos coletores das cadeias ilíacas externas e comuns e para aórticas abdominais. A nível de $\mathrm{T}_{12}-\mathrm{L}_{1}$ observou-se interrupção do ducto torácico. Na fase vascular havia a passagem de meio de contraste ao mediastino não se observando imagem de pertuito condicionando o extravasamento (fig. 3). As radiografias de $24 \mathrm{~h}$ não evidenciaram estase linfática em ductos 


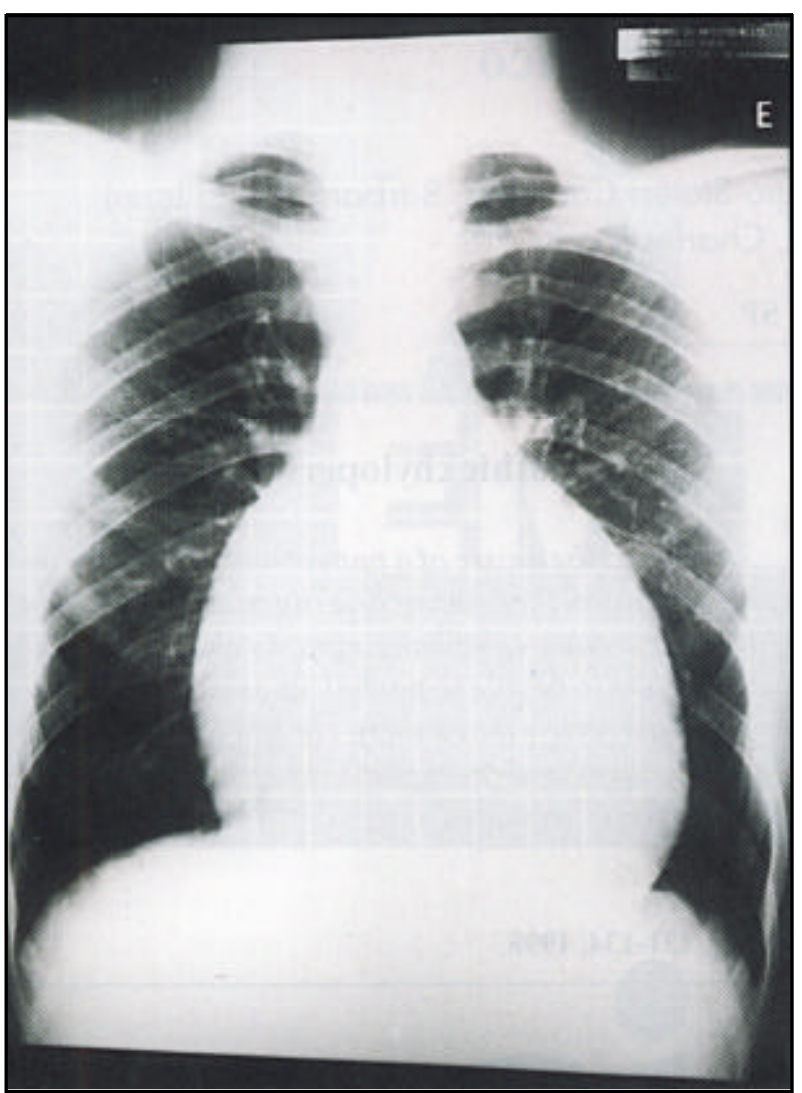

Fig. 1 - Radiografia de tórax em projeção PA mostra aumento da área cardíaca.

coletores. No tórax observaram-se remanescentes de pequenas quantidades de meio de contraste no mediastino e cavidade pericárdica. A tomografia abdominal não mostrou adenomegalias ou processos que justificassem a obstrução do ducto.

Na evolução após retirada do dreno pleural direito evidenciaram-se pneumopericárdico importante e pneumotórax à direita, tendo sido o paciente submetido a nova drenagem pleural à direita no $7^{\circ}$ dia de pós-operatório. $\mathrm{O}$ paciente evoluiu com derrame pleural à direita. $\mathrm{O}$ débito pelo dreno pleural permaneceu elevado por 40 dias (média = 237,5mL/dia) com líquido de aspecto branco leitoso. A análise bioquímica do líquido pleural revelou altos níveis de triglicérides (384mg/dL). Devido à manutenção do elevado débito pelo dreno foi introduzida dieta hipogordurosa com triglicérides de cadeia média (TCM) por 30 dias, sem resultado satisfatório. A outra opção utilizada como tratamento foi a introdução de nutrição parenteral total por sete dias, também sem resultado significativo em relação ao débito do quilo. Havendo obstrução do dreno pleural, optou-se pela sua retirada, tendo o paciente evoluindo com pneumotórax à direita. Dessa forma, optou-se por nova introdução de dieta hipogordurosa e acompanhamento do derrame pleural, que se manteve inalterado durante a internação. $O$ paciente recebeu alta em bom estado geral, com derrame pleural pequeno à direita (fig. 4), com dieta hipogordurosa. Após três meses de acompanhamento ambulatorial o paciente mantém-se estável, não se observando aumento do derrame pleural.

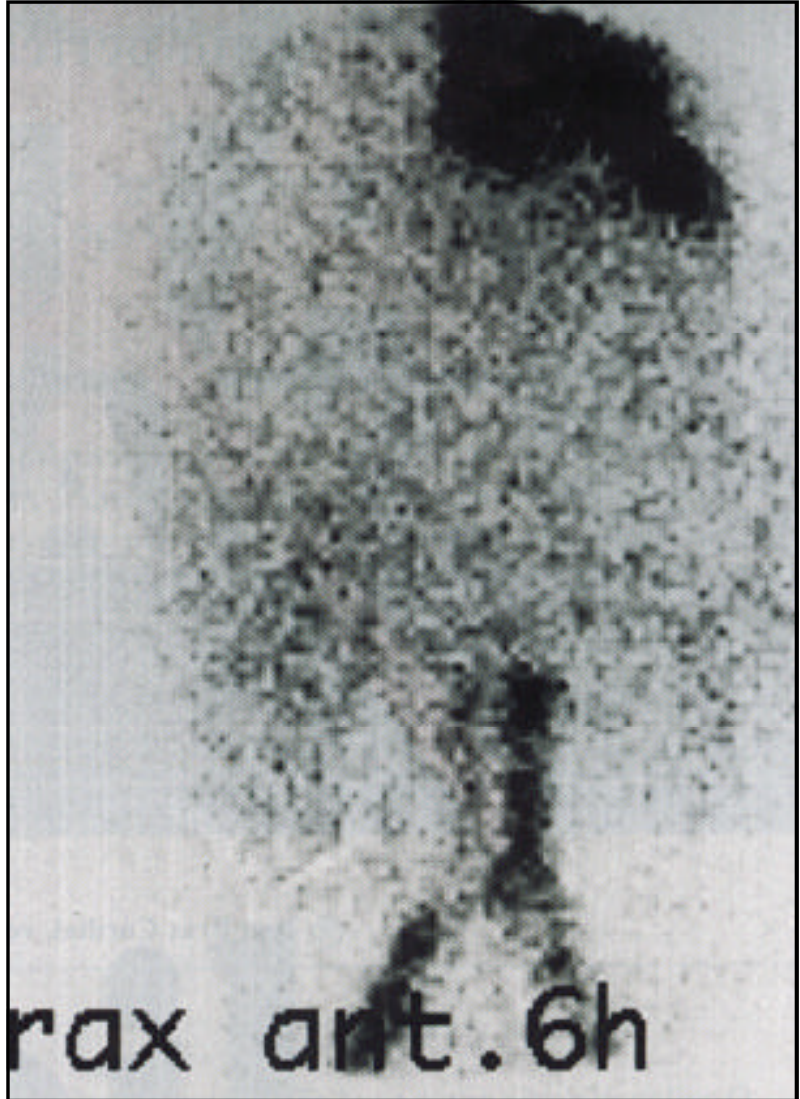

Fig. 2 - Linfocintilografia com macroagregados de albumina marcados com tecnécio99 demonstrando aplasia do ducto torácico em sua porção distal e presença do contraste no mediastino.

\section{Discussão}

Quilopericárdio é um acúmulo de quilo no espaço pericárdico. Denomina-se quilo quando a linfa apresenta altos teores de gordura e proteínas. A linfa é conduzida da periferia de tecidos e intestinos ao sistema vascular, inicialmente por pequenos canalículos e, a partir daí, até a cisterna magna e ducto torácico. Toda a linfa no organismo segue este trajeto desembocando na veia inominada junto à desembocadura na veia jugular interna. A produção diária de linfaé ao redor de 1,5 a $2,0 \mathrm{~L}^{3}$.

A etiologia do quilopericárdio pode ser decorrente de traumas, pancreatites, cirurgias torácicas, linfangiomatose congênita, secundária a obstruções no ducto torácico ou na drenagem na veia subclávia esquerda, por neoplasias e tuberculose, e mesmo associada a síndromes congênitas, como na síndrome de Noonan ${ }^{4,5}$.

A primeira descrição de quilopericárdio isolado foi feita por Hasebrock, em 1888, que descreveu 22,6cc de líquido quiloso removido de necropsia. Em 1954, Groves e Effer descreveram o quilopericárdio primário como sendo acúmulo de quilo no pericárdio de etiologia não definida. Yutzel e col${ }^{2}$ relataram, em 1997, somente 89 casos na literatura de quilopericárdio primário idiopático, denotando ser uma entidade clínica bastante infreqüente.

A fisiopatologia para o acúmulo de quilo no espaço pericárdico pode ser: devido a comunicação linfopericárdio 


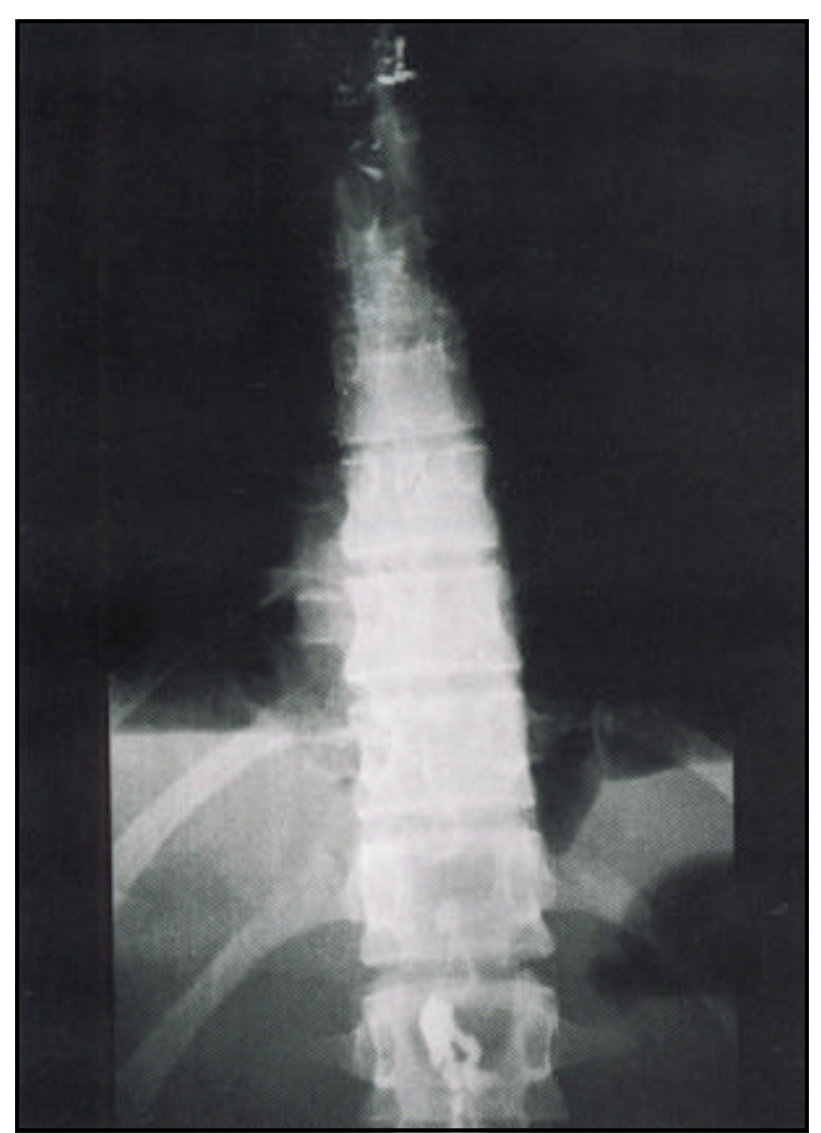

Fig. 3 - Linfografia demonstrando ao nível de T12-L1 interrupção do ducto torácico, não se visibilizando sua porção distal.

direta, com presença de fistulas ${ }^{1,6}$, decorrente de refluxo de quilo por hipertensão linfática, ocasionando perda do papel valvular e falência em estabelecer drenagem linfática colateral e secundária a aumento da permeabilidade das paredes dos vasos linfáticos ${ }^{1}$.

A idade média de aparecimento é ao redor de 23 anos, porém, há casos descritos desde o nascimento até 63 anos, sendo mais prevalente no sexo masculino. Os sintomas de apresentação clínica variam desde paciente assintomático até casos de tamponamento cardíaco, sendo este último bastante infreqüente. O quilopericárdio idiopático, raramente, está associado a quilotórax idiopático.

O diagnóstico clínico de derrame pericárdico é feito, geralmente, pela radiografia simples de tórax, demonstrando área cardíaca aumentada, eletrocardiograma com complexos de baixa voltagem e ecocardiograma. O diagnóstico de quilopericárdio baseia-se, na pericardiocentese, no encontro de líquido quiloso, confirmado pela presença de quilomícrons e elevados níveis de triglicérides ${ }^{7}$.

Uma vez feito o diagnóstico de quilopericárdio, devese realizar o estudo anatômico do ducto torácico através da linfografia. A linfografiaé um exame complementar de importância diagnóstica e fundamental para a definição da estratégia terapêutica, devido às variações do ducto torácico. Cha e Sirijiintakarn ${ }^{8}$ descreveram variação na anatomia do ducto torácico em $23 \%$, duplicado em $32 \%$, formação de plexo segmentar em $56 \%$ e de múltiplos plexos em 19\%.En-

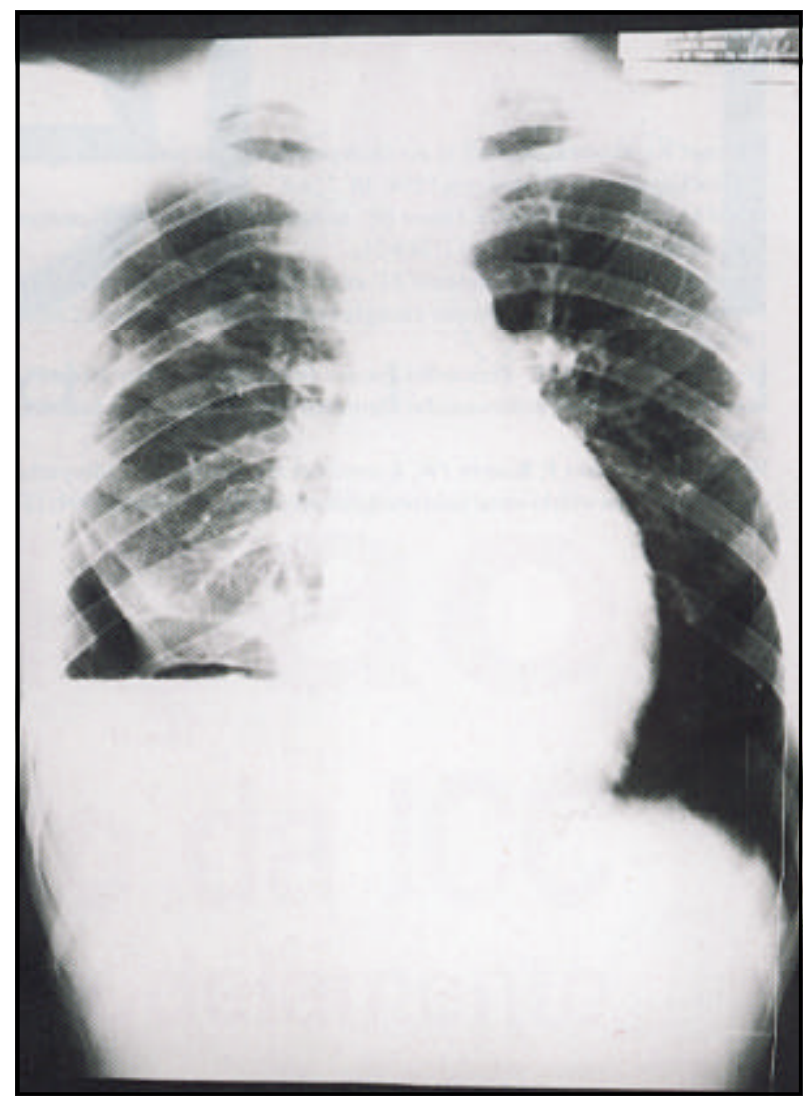

Fig. 4 - Radiografia de tórax projeção PA mostrando pequeno derrame pleural à direita e pneumotórax encistado.

tretanto, nesse estudo não esteve presente nenhum caso de quilopericárdio.

A linfografia também pode auxiliar no encontro de fístulas linfático-pericárdicas, transudação linfática e, em nosso caso específico, demonstrar aplasia do ducto. Mewis e $\operatorname{col}^{9}$, à semelhança de nosso caso, descreveram um paciente com quilopericárdio primário devido a aplasia parcial do ducto torácico ao nível da $12^{a}$ vértebra torácica.

Com relação à terapêutica do quilopericárdio, a primeira opçãoé a dieta hipogordurosa. Utilizam-se TCM, cuja finalidade é diminuir a formação de linfa. Sabe-se que a absorção dos TCM é feita via veia porta, diferentemente dos triglicérides de cadeia longa, que passam por um processo de esterificação e formação de quilomícrons que são absorvidos por via linfática. Com isso, a formação de linfa seria diminuída e o derrame tenderia a desaparecer ${ }^{10}$. Infelizmente essa terapêutica não é eficaz nos casos de quilopericárdio espontâneo ${ }^{7}$, sendo empregada nos casos de quilopericárdio traumáticos com bons resultados ${ }^{3}$.

Outra opção terapêutica que pode ser utilizada é o jejum e a nutrição parenteral prolongada, porém, nos casos primários também não chega a ser eficaz. Assim, nos casos refratários, o tratamento cirúrgico é o mais indicado, procurando-se realizar a ligadura do ducto torácico acima do diafragma, combinado com pericardiectomia parcial. A pericardiectomia é utilizada para realizar drenagem completa e evitar evolução para pericardite constritiva ${ }^{7}$. 


\section{Referências}

1. Martinez JG, Marco E, Marín F et al - Quilopericárdio tras pericarditis aguda. Revista Española de Cardiologia 1996; 49: 226-8.

2. Yutzel M, Yidizeli B, Zonuzi F, Hasan BF - Isolated primary chylopericardium. Eur J Cardiothorac Surg 1997; 12: 319-21.

3. Jatene BF, Bosisio IBJ, Monteiro AC et al - Quilotórax pós-traumático. Experiência no pós-operatório de cirurgia cardiotorácica. Arq Bras Cardiol 1993; 61: 229-32.

4. Lorell BH, Braunwald E - Pericardial disease. Braunwald E, editor. Heart Disease. A Textbook of Cardiovascular Medicine. Philadelphia: WB Saunders, 1992: 1505.

5. Winter RJ, Bresser P, Romer JW, Kromhout JG, Reekers J - Idiopathic chylopericardium with bilateral pulmonary reflux of chyle. Am Heart J 1994; 127:
936-9.

6. Samaniego V, Moguel R, Meaney E, Alvarez-Toatado R, Castillon M - Massive primary chilopericardium due to congenital fistulas. Am Heart J 1993; 126 : 1005-6.

7. Svedjeholm R, Jansson K, Olin C - Primary idiopathic chylopericardium- a case report and review of the literature. Eur J Cardio-Thoracic Surg 1997; 11:387-90.

8. Cha EM, Sirijiintakarn P - Anatomic variation of the thoracic duct and visualization of mediatinal lymph nodes. Radiology 1976; 119: 45-8.

9. Mewis C, Kuhlkamp, Sokiranski R, Karsch KR - Primary chylopericardium due to partial aplasia of the thoracic duct. Eur Heart J 1997; 18: 880-1.

10. Diógenes MSB, Carvalho VB, Rozov T et al - Derrame pericárdico devido à linfangiectasia pulmonar. Arq Bras Cardiol 1988; 5: 185-92. 